\title{
Solar Nanoantennas energy based characterization
}

\author{
C. Di Garbo ${ }^{1}$, P. Livreri ${ }^{1}$ and G. Vitale ${ }^{2}$ \\ ${ }^{1}$ Dipartimento di Energia, ingegneria dell'Informazione, e modelli Matematici, Università di Palermo, Viale delle Scienze, \\ Ed.9, 90128 Palermo (Italy), email: chiara.digarbo(at)unipa.it, patrizia.livreri(at)unipa.it \\ ${ }^{2}$ Istituto di Studi sui Sistemi Intelligenti per l'Automazione (ISSIA), Consiglio Nazionale delle Ricerche (CNR), Via Dante \\ Alighieri, n. 12 - 90141Palermo (Italy), corresponding author, email: gianpaolo.vitale(at)cnr.it
}

\begin{abstract}
Solar nanoantennas appear a very interesting solution to exploit solar radiation, as a matter of fact, their operating principle allows heat losses to be lessened compared to traditional photovoltaic cells and efficiency to be increased. Anyway, some technological issues are to be overcome. Literature often proposes a characterization by radiation efficiency typical of traditional antennas. Since the aim of solar nanoantennas is to harvest energy, in this paper the most promising devices are characterized by the upper bound of deliverable power density calculated in the whole operating wavelengths range.
\end{abstract}

\section{Key words}

Nanoantenna, photovoltaic cell, energy conversion.

\section{Introduction}

In the last decade, the increasing demand for free and clean energy had led to the development of alternative and renewable energy sources, like, for example, solar photovoltaic (PV) cells. They are pn junction cells based on the absorption of photons whose energy is greater than the bandgap; as a consequence electrons from the valence band to the conduction band are excited. Solar radiation is composed of energy photons whose energy is generally higher than the bandgap of the solar cell, as a consequence, the excess of energy is converted into heat by lattice vibrations or phonons. For this reason, the upper efficiency is about 20\%. In order to overcome this limitation, multi junction PV cells have been devised, anyway it does not seem a cost-effective solution. Another drawback is the strong dependence on daylight and, consequently, on weather conditions [1-2]. Thus, an alternative approach is needed. In this context, optical antennas or nanoantennas seem the best candidates to replace the solar cells. Similar to traditional RF antennas, nanoantennas capture the incident electromagnetic wave (visible light or infrared) causing an AC current onto the antenna surface such that it oscillates at the same frequency of that wave. The movement of the electrons produces an alternating current in the antenna circuit. Since the aim of these devices is to supply an external load, the AC current has to be rectified by a suitable diode [3-4]. It can be noted that this is a difference in comparison to PV cells that provide a DC current; whereas in both systems there is need to match the source with the load to achieve the maximum available power [5-10].

Since the wavelengths in the solar spectrum range from about $300 \mathrm{~nm}$ to $2000 \mathrm{~nm}$, a length of the nanoantenna of the order of hundreds nanometers is expected. Finally by this feature the infrared (IR) region of the electromagnetic spectrum can be exploited as well [4]. Two main types of nanoantennas can be recognized: metallic and dielectric [11].

Usually, nanoantennas are characterized by the radiation efficiency versus wavelength. In this paper some representative nanoantennas are compared by calculating the maximum power density available at the terminals since it is the quantity of interest for energy harvesting purposes.

\section{Metallic nanoantennas}

A metallic nanoantenna consists of one or more metallic nanoparticles working in a range of frequencies close to plasmonic resonance of the used metal. This phenomenon occurs when metallic nanoantennas absorb the incident electromagnetic radiation at the resonant frequency, thus inducing a cyclic plasma movement of free electrons and generating alternating current, which then flows toward the antenna feed point. Thus, the electric field is concentrated at the center of this point [12]. The metals mainly used are gold, silver, aluminum and copper, whose plasmonic properties lie in the optical range. In the literature a great variety of metallic nanoantennas depending on the geometry of nanoparticles can be found. The simplest type of metallic nanoantenna is the monopole nanoantenna. It consists of a single metallic nanoparticle that increases the strength of the electromagnetic field in its vicinity.

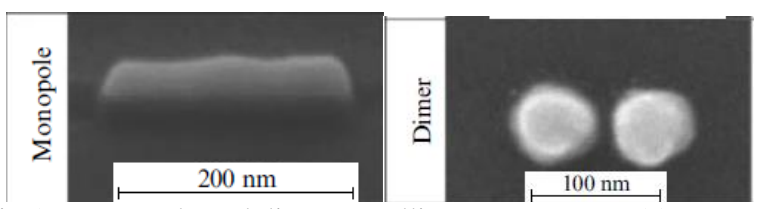

Fig.1. Monopole and dimer metallic nanoantennas [11].

The dimer nanoantennas confine the high field in the gap between two metallic nanoparticles and include dipole nanoantennas and 'bowtie' nanoantennas. The dipole 
nanoantennas consist of two coupled nanorods or nanospheres. They offer several advantages: easy production, fine tuning and high confinement of electric field in the gap [11]. Figure 1 shows a monopole and a dimer antennas. A bowtie nanoantenna consists of two triangles facing each other from their apices and with a gap in between. The electric field is typically concentrated at its gap [11,13]. Such a nanoantenna has a broad bandwidth and a lower confinement factor due to a larger amount of metal used for its fabrication. Consequently, it exhibits greater dissipative losses. Moreover, with this type of nanoantenna it is possible to construct an array by coupling several bowtie elements in one configuration and by combining the electric field from each element at the array feeding point, where a rectifier can be embedded [11, 13-14]. Dipole and Bowtie nanoantennas are shown in figure 2.

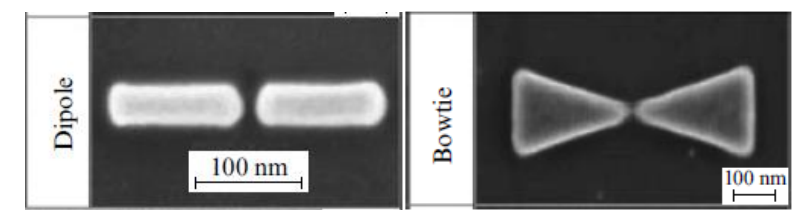

Fig. 2. Dipole and Bowtie metallic nanoantennas [11].

In order to obtain radiation toward the desired direction, Yagi-Uda nanoantennas are used as sketched in figure 3. These devices consist of a reflector and one or several directors. They are narrowband devices that work at frequencies close to those of the plasmon resonances, determined by the dimensions of the antenna elements [11],[15]

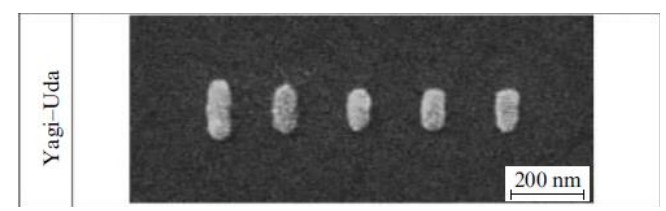

Fig. 3. Metallic Yagi-Uda nanoantennas [11].

Spiral antennas reveal planar structure, circular polarization and broadband characteristics. They are good resonators and they can produce a large field at the feed point. It is possible to enhance the performance of this nanoantenna by increasing the number of arms and, consequently, its aperture area is increased [13]. A square Spiral nanoantennas is represented in figure 4.

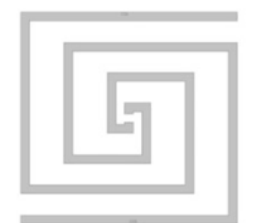

Fig. 4. Square Spiral nanoantenna [13].

The output of a nanoantenna is an AC signal, hence, a proper diode-based rectifier, typically an ultra-high speed diode, and a Low Pass (LP) filter are used to produce DC power [13]. The block diagram of a typical so-called solar rectenna is shown in figure 5 [13-18].

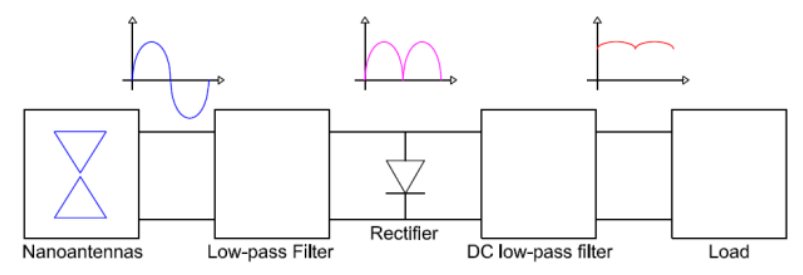

Fig.5: Block diagram of solar rectenna

\section{Dielectric nanoantennas}

The main drawback of metallic nanoantennas is the large dissipative losses due to utilized metal that reduce its range of working frequencies and result in low radiation efficiency [19]. To overcome this disadvantage a new type of nanoantennas based on dielectric nanoparticles with a high index dielectric constant has been realized. Dielectric nanoantennas are fabricated from optically transparent materials and exhibit smaller dissipative losses in the material with enhanced magnetic response in the visible. They support simultaneously both electric and magnetic resonances, which can be controlled independently [19]. They show a resonant response due to the formation of an effective resonator inside the particle. The dielectric nanoantennas include also the antennas based on semiconducting particles, because this type of materials are sufficiently transparent in the visible frequency range. Typically dielectric nanoantennas consist of spherical particles and sometimes of rods and disks [11]. Whispering gallery antennas consist of dielectric microspheres and possess good directional capabilities by virtue of their large surface area. It is possible to consider Huygens optical element for the creation of totally dielectric nanoantennas. Excitation of such a dielectric particle induces electric and magnetic dipole moments. Depending on the frequencies and on the material and the size of the nanoparticles, the amplitudes of electric and magnetic polarizabilities can be equal to each other. In this way, it is possible to create compact fully dielectric nanoantennas with good directional properties. Another type of dielectric nanoantenna is the dielectric Yagi-Uda nanoantenna. It consists of more dielectric nanoparticles of equal size. It exhibits the radiation efficiency that slowly decreases with decreasing distance between the elements [11]. This distance influences the operational regime of a dielectric Yagi-Uda nanoantenna. In order to have the best directivity, this type of nanoantenna has to operate in a magnetic regime [11]. In order to overcome the drawbacks of metallic and dielectric nanoantennas a hybrid nanoantenna has been realized. This consists of a pair of metallic nanoparticles that enhances the spontaneous emission rate of an emitter placed between them, and a dielectric ( $\mathrm{TiO} 2$ ) microsphere needed to effectively collect radiated light into a narrow beam [11].

\section{Delivered power evaluation}

In order to evaluate the energy that can be delivered by a nanoantenna, the Sun has been characterized by a blackbody source with temperature equal to $5778 \mathrm{~K}$. Energy is 
radiated uniformly in all directions following the Plank's blackbody radiation formula:

$$
W_{\lambda}=\frac{2 \pi h c^{2}}{\lambda^{5}\left(e^{\frac{h c}{\lambda k T}}-1\right)}
$$

Where $\mathrm{h}$ is the Plank's constant equal to $6.6310^{-34}[\mathrm{~J} \mathrm{~s}]$ and $\mathrm{k}$ is the Boltzmann's constant equal to $1.3810^{-23}[\mathrm{~J} / \mathrm{K}]$. It should be noted that (1) is expressed in $\left[\mathrm{W} / \mathrm{m}^{2} / \mathrm{unit}\right.$ wavelength in meters], its shape depends on temperature and wavelength. The total energy emitted by Sun can be obtained multiplying (1) for the Sun surface. Since energy coming from the Sun travels for about $150 \cdot 10^{6} \mathrm{~km}$ before encountering the Earth's atmosphere, remaining practically unchanged, the energy on the Earth is obtained by dividing for the area of the surface whose radius is the distance Sun-Earth. Since the surface of the Sun is given by $\pi$. (Sun_radius) ${ }^{2}$ and the surface of the sphere having the Sun as center tangential to the Earth is $\pi$. (distance_Sun_Earth) $^{2}$, eq. (1) must be scaled of a coefficient equal to $\mathrm{C}=2.164 \cdot 10^{-5}$. Integrating (1) over the whole wavelengths interval, the solar constant of 1.3661 $\mathrm{kW} / \mathrm{m}^{2}$ is obtained. The spectrum of solar radiation calculated at the Earth surface is drawn in figure 6. [2, 20] It should be noted that the maximum of the spectrum equals about $500 \mathrm{~nm}$.

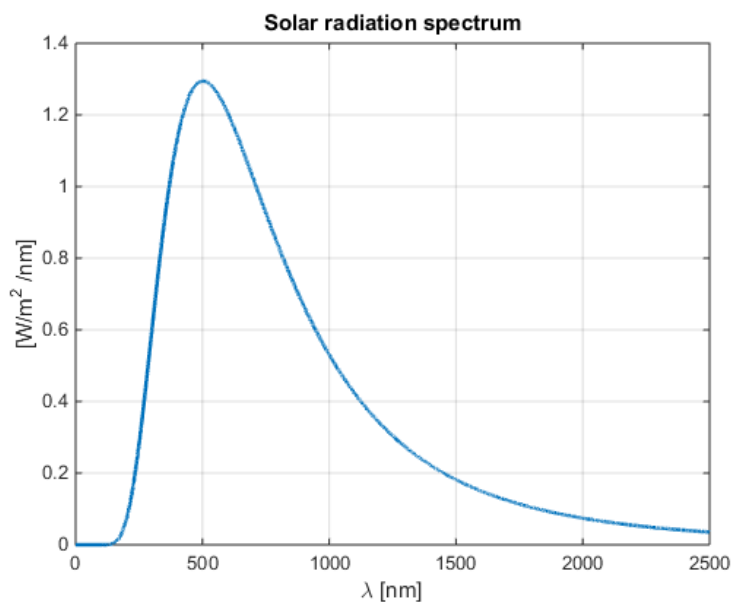

Fig. 6. spectrum of the solar radiation for $\mathrm{T}=5778$, calculated at the Earth surface.

Inside the atmosphere a part of sunlight is absorbed from molecules that raise their own energy. As a consequence, some wavelengths are lessened and the curve of figure 6 actually presents some hole known as absorption lines. The presence of ozone produces absorption in the ultraviolet region while water vapor and carbon dioxide absorb mainly in the visible and infrared parts of the spectrum.

Scattered sunlight makes blue the sky color and gives us sunlight from north when Sun is in the south. Without sunlight scattering, the sky would appear black as during the night with the moon. Even with a light shadowing, sunlight is scattered and these rays cannot be focused as the direct rays. Finally, at ground level, sunlight can be either absorbed or reflected.
The path through atmosphere plays an important role in the spectrum of sunlight at Earth level. Conventionally the vertical path directly to the sea level is defined as unitary air mass (AM1). If Sun rays are inclined, the AM value is greater than one. Considering the absorption at AM1, the power density coming from the Sun is $70 \%$ of the power density at the atmosphere surface. It is slightly greater than $1000 \mathrm{~W} / \mathrm{m}^{2}$. For AM different from unity, the power density $\mathrm{P}\left[\mathrm{W} / \mathrm{m}^{2}\right]$ can be deduced by:

$$
P=1367(0.7)^{A M^{0.678}}
$$

The value of AM1.5 is used as a standard calibration spectrum for photovoltaic cells. It corresponds to the spectrum obtained when the Sun forms an angle of $48.19^{\circ}$ with the zenith. Figure 7 shows different values of AM.

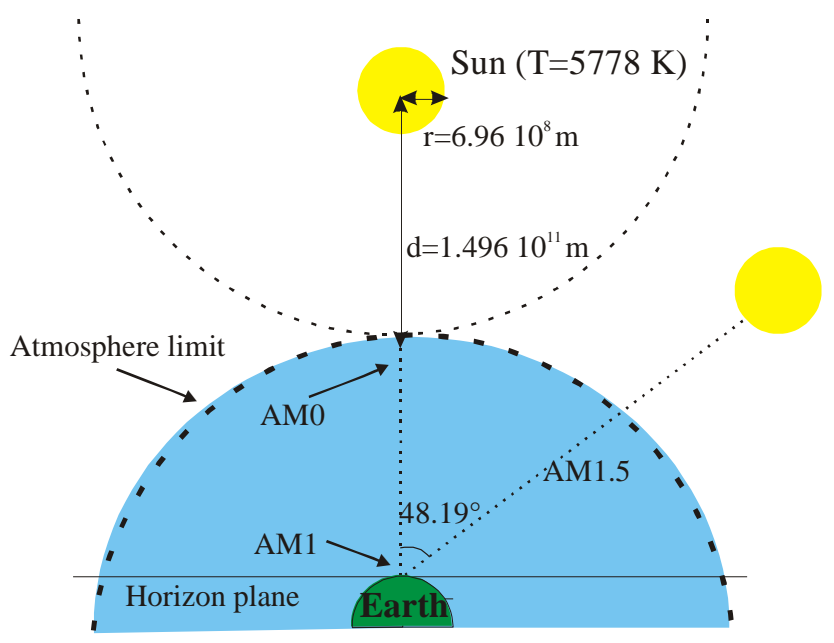

Fig.7. Air Mass conditions

If photovoltaic performance are evaluated under conditions that differs from AM1.5, a calibration factor must be adopted. In general this correction factor gives the short-circuit current taking into account environmental conditions as AM and water vapour and turbidity. It should be borne in mind that, in a traditional semiconductor solar cell, the calibration factor value is influenced by the semiconductor used to set up the photovoltaic generator, for example amorphous silicon has a much higher sensitivity to water and turbidity than crystalline $\mathrm{Si}, \mathrm{CdTe}$ or $\mathrm{CuInSe} 2$. In any case the calibration factor is more accurate for conditions near to AM1.5 [1,2].

Nanoantennas are characterized by the radiation efficiency whose value depends on the wavelength

$$
\eta^{\text {rad }}=\frac{P^{\text {rad }}}{P^{\text {inject }}}=\frac{P^{\text {rad }}}{P^{\text {rad }}+P^{\text {loss }}}
$$

Finally, the power density delivered by a nanoantenna can be calculated by: 


$$
P_{t o t}=\int_{\lambda \min }^{\lambda \max } W_{\lambda}(\lambda, T) \eta^{r a d}(\lambda) d \lambda
$$

Where the interval $\lambda_{\min }-\lambda_{\max }$ is the operating antenna range.

It must be taken into account that the power calculated by (3) represents the upper limit. Three main factors contribute to lessen this value. They are: a) the coupling efficiency between the antenna and the diode, b) the rectification efficiency and c) the coupling efficiency between the diode and the load. The success of nanoantennas is also tied to the resolution of these technological issues which are described in the following section.

\section{Technological issues}

As above mentioned, the nanoantenna is not able to convert the high frequency collected power dierctly in DC power. As a consequence, the rectenna system needs to employ a rectifier, typically an ultra-high speed diode $[12,17,18]$. This device has to respect many requirements imposed for optical frequency rectification, like high responsivity, small size, small turn on voltage and efficient performance at $\mathrm{THz}$ e IR frequencies. Moreover, these types of rectifier should be coplanar and coupled to the nanoantenna. The equivalent circuit of a solar rectenna consists of the rectifier in parallel with the nanoantenna circuit. The equivalent scheme is shown in figure 8 .

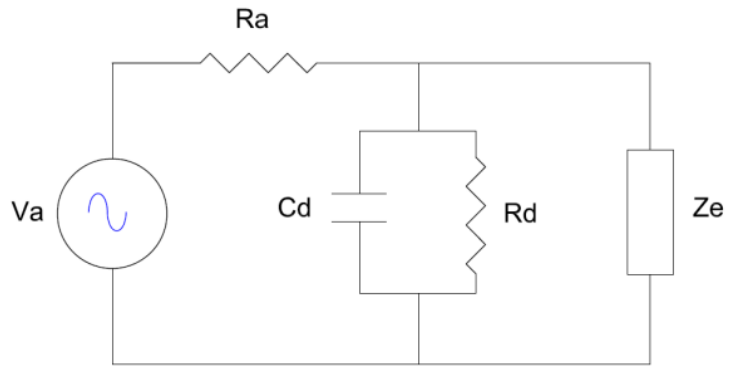

Fig.8: Equivalent circuit of a solar rectenna

In order to obtain a DC signal, the conversion efficiency of a rectenna is conditioned by the power loss in the diodes, by the impedence matching between the nanoantenna and the rectifier and between the rectifier and the load, and by the nanoantenna efficiency [12]. Relatively to the rectifier, the main problems regard diode resistance, capacitance, and reverse-bias leakage. To obtain an efficient power transfer, the coupling between the impedance of the diode and the impedance of the nanoantenna is required. The latter is usually characterized by the resistance in a range from few hundreds to thousand ohms at visible light frequencies and lower at terahertz frequencies [3]. Thus, to improve the power transfer, the diode resistance has to be sufficiently low and close to the nanoantenna resistance. In this way a significant reduction of power loss is achieved. The diode capacitance gives a significant contribution to its switching time. In order to have a fast diode, the value of this capacitance has to be small. Another problem is the significant current for negative voltages in the diode. This current is required to be lower, on the order of $1 \mu \mathrm{A}$ or less.
This represents the main challenge to achieve a diode for optical frequency rectification [3]. A low-pass filter is placed between the nanoantenna and the rectifier. The aim of this device is to avoid that the radiation of higher harmonics, generated from the rectification of the non linear diode, comes back to the nanoantenna resulting in power losses. Furthermore, this filter makes possible the matching between the nanoantenna and the following circuitry [13]. To allow the maximum signal transfer to the load, the impedance matching between the rectenna system and the load is required. The maximum power transfer is reached when the load impedance is the conjugate of the rectenna impedance. In order to separate the high-frequency component from the DC signal a DC low-pass filter is placed between the rectifier and the load [12]. The main candidate of an ultra-high speed diode for rectification, that could operate at petahertz frequencies, is Metal-Insulator-Metal diode (MIM diode). This is a thin film device that consists of an insulator layer of few nanometers in thickness placed between two metal electrodes [13, 18]. This diode has a carrier transit time of the order of femtosecond and can be easily integrated with the nanoantenna. It exhibits a large $\mathrm{RC}$ time constant and can work efficiently at low terahetz frequencies. Other types of diodes taken into account for rectification at higher frequencies are: the Metal-Multi insulator-Metal diodes, that have a greater responsivity, and the MIM Travelling-Wave diodes, that present a higher matching efficiency between nanoantenna and rectifier [3].

\section{Results}

In order to compare the performance of some nanoantennas proposed in literature, they have been characterized considering the upper limit of the deliverable energy density given by (3).

The following nanoantennas are analysed in the following: a) dipole, b) dielectric vs. metallic Yagi-Uda, c) Yagi-Uda, d) bowtie

\section{A. Dipole antenna}

Figure 8 shows the efficiency versus wavelength of silver dipole antennas characterized by a length from $100 \mathrm{~nm}$ to $350 \mathrm{~nm}$. These curves are taken from [21].

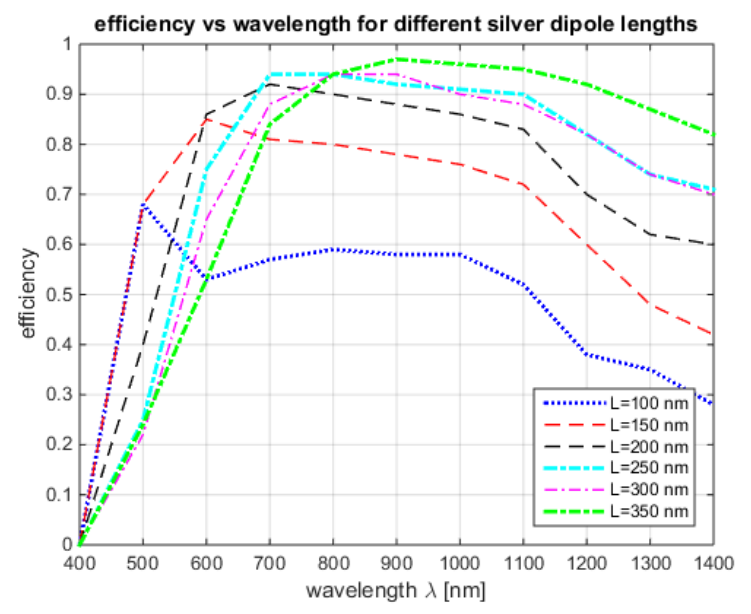

Fig.8. Efficiency of different silver dipole antennas 
It can be noted that the dipole length influences the efficiency curve. In particular, when the dipole length is increased the curve increases the maximum value of its efficiency but it is shifted towards right becoming far from the peak of solar radiation spectrum (about $500 \mathrm{~nm}$ ).

The upper bound of the available power density is summarized in table I. Despite the differences among the curves of figure 8 , it can be noted that the power density shows a small variations for nanoantennas with length from $150 \mathrm{~nm}$ to $350 \mathrm{~nm}$.

Table I

Power density values for dipole nanoantennas

\begin{tabular}{|c|c|}
\hline Dipole length [nm] & $\begin{array}{c}\text { Power density } \\
{\left[\mathbf{W} / \mathbf{m}^{\mathbf{2}}\right]}\end{array}$ \\
\hline 100 & 379.49 \\
\hline 150 & 505.59 \\
\hline 200 & 517.97 \\
\hline 250 & 508.73 \\
\hline 300 & 486.39 \\
\hline 350 & 488.16 \\
\hline
\end{tabular}

\section{B. Dielectric vs. metallic Yagi-Uda antennas}

Figure 9 shows the efficiency versus wavelength of a dielectric and a metallic antennas characterized by a separation distance $\mathrm{D}=70 \mathrm{~nm}$. These curves are taken from [19].

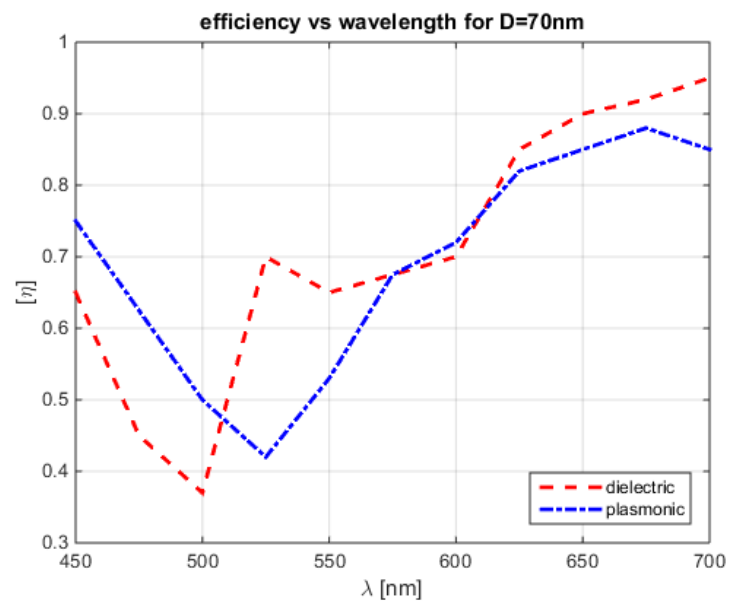

Fig.9. Efficiency curves of dielectric and plasmonic antenna

The upper bound of the available power density is summarized in table II. It can be noted that the delivered power is lower compared with silver dipole antennas and that even in this case the obtained values do not differ appreciably each other. In [19] some other curves with lower values of $D$ are proposed but the delivered energy is lower than the obtained one for $\mathrm{D}=70 \mathrm{~nm}$.

Table II

Power density values for plasmonic and dielectric nanoantennas

\begin{tabular}{|c|c|}
\hline antenna & $\begin{array}{c}\text { Power density } \\
{\left[\mathbf{W} / \mathbf{m}^{2}\right]}\end{array}$ \\
\hline plasmonic & 225.79 \\
\hline dielectric & 230.95 \\
\hline
\end{tabular}

It can be noted that the upper bound of the deliverable power density is nearly the same.

\section{Yagi-Uda antennas}

The efficiency curves of totally dielectric Yagi-Uda nanoantennas for different values of the distance $G$ between the director surfaces studied in [19] are drawn in figure 10. In this case it is possible to note that decreasing the distance between the elements, the radiation efficiency slowly decreases. The power density values are shown in table III.

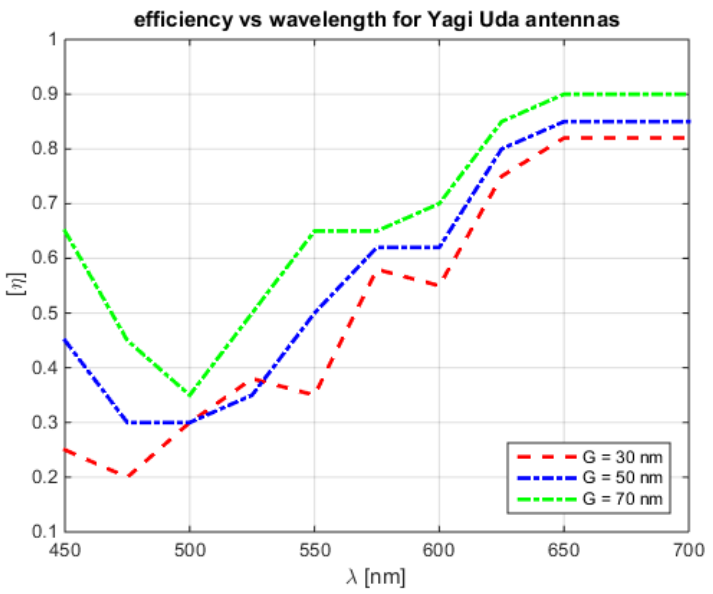

Fig.10. Efficiency curves of dielectric Yagi-Uda antennas

Table III

Power density values for Yagi-Uda nanoantennas

\begin{tabular}{|c|c|}
\hline $\begin{array}{c}\text { Yagi Uda } \\
\text { G [nm] }\end{array}$ & $\begin{array}{c}\text { Power density } \\
{\left[\mathbf{W} / \mathbf{m}^{\mathbf{2}}\right]}\end{array}$ \\
\hline 30 & 169.64 \\
\hline 50 & 190.14 \\
\hline 70 & 230.95 \\
\hline
\end{tabular}

\section{Bowtie antenna}

In [21] the radiation efficiency versus wavelength of the $\mathrm{Au}$ modified bowtie nanoantennas is proposed. This antenna is characterized by polinomial sides; $\alpha$ is the polynomial order of the sides variation as shown in figure 11. The efficiency is calculated for different values of $\alpha$ $(\alpha=1,2,3,4)$ and for $\mathrm{L}=150 \mathrm{~nm}$ and $\mathrm{d}=10 \mathrm{~nm}$, where $\mathrm{L}$ is the side length of conventional bowtie triangle and $\mathrm{d}$ is the gap. It can be noted that there is a shift of resonance wavelengths to the right for high values of $\alpha$; moreover, the maximum of efficiency decreases for large values of $\alpha$. The efficiency curves versus wavelength are sketched in figure 12 where, in general, a lower efficiency, compared to the other antennas can be appreciated. The power density values are summarized in table IV. 


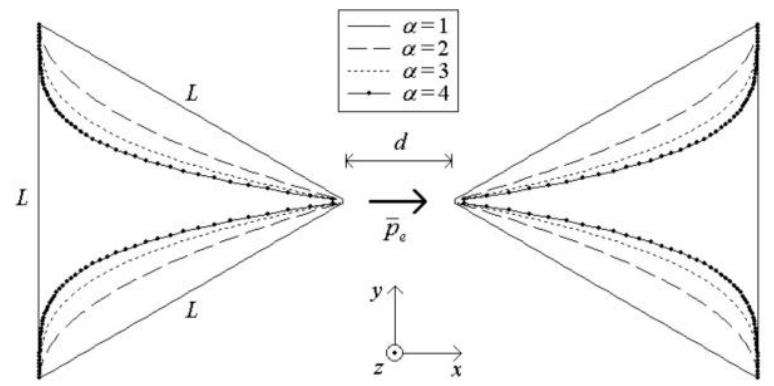

Fig.11. Polinomial bowtie antennas

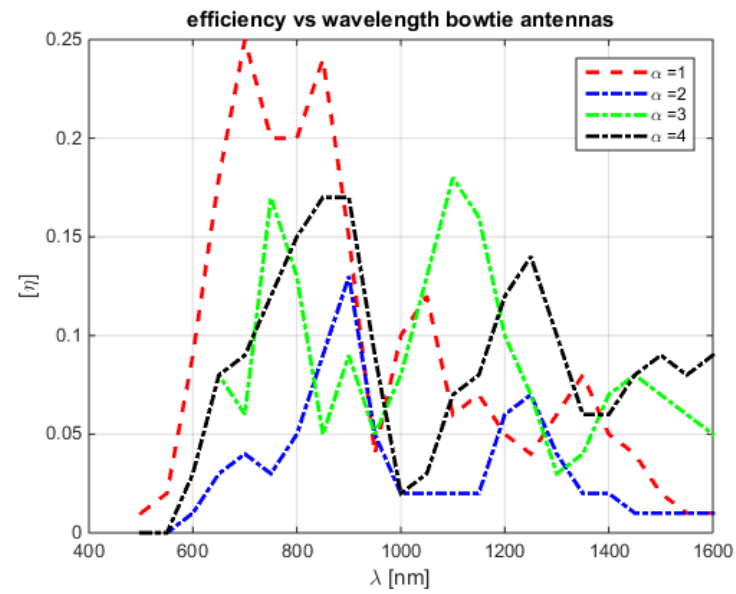

Fig.12. Efficiency curves of bowtie antennas

Table IV

Power density values for bowtie nanoantennas

\begin{tabular}{|c|c|}
\hline $\begin{array}{c}\text { Bowtie antenna } \\
(\boldsymbol{\alpha})\end{array}$ & $\begin{array}{c}\text { Power density } \\
{\left[\mathbf{W} / \mathbf{m}^{\mathbf{2}}\right]}\end{array}$ \\
\hline 1 & 75.58 \\
\hline 2 & 22.22 \\
\hline 3 & 47.77 \\
\hline 4 & 51.50 \\
\hline
\end{tabular}

\section{Conclusions}

Solar nanoantennas features have been analysed in this paper. The upper bound of the available power density has been calculated for some antennas presented in literature on the basis of their efficiency. Best results are exhibited by dipole antennas. Anyway, the obtained values represent the upper limit of the achievable energy since there is need of an optimal matching with the rectifier and the load. Despite some technological issues regarding mainly the coupling components between the nanoantenna and the load are to be overcome, they exhibits a power density greater than traditional PV solar cells and can help the energy harvesting process contributing to the lessening of the global warming in the next future.

\section{References}

[1] Di Piazza M. C., Vitale G. 'Photovoltaic Sources: Modelling and Emulation', Springer, 2013, ISBN: ISSN 1865-3529 ISSN 1865-3537, DOI 10.1007/978-1-4471-4378-9.
[2] S. Wentham, M. A. Green, M. E. Watt, R. Corkish, "Applied photovoltaics", earthscan, 2009.

[3] G. Moddel "Will Rectenna Solar Cells be practical", chapter 1, Rectenna Solar Cells, Springer, pp.3-24, (2013).

[4] G. Sadashivappa, N. P. Sharvari, "Nanoantenna - a review", International journal of Renewable Energy Tecnology Research, vol. 4, n. 1, Jan 2015, pp 1-9.

[5] M. C. Di Piazza, M. Pucci, A. Ragusa, G. Vitale "Fuzzified PI Voltage Control for Boost Converters in Multi-String PV Plants", 34th Annual Conference of the IEEE Industrial Electronics Society, IECON-2008. 10-13 November 2008.

[6] Marian, V.; Allard, B.; Vollaire, C.; Verdier, J., "Strategy for Microwave Energy Harvesting From Ambient Field or a Feeding Source", IEEE Transactions on Power Electronics, 2012, Vol. 27, Issue: 11, pp: 4481 - 4491

[7] L. Novotny, N. van Hulst "Antennas for light" Nature Photonics 5, 83-90 2011.

[8] V. Giannini, A. I. Fernandez-Domínguez, S. C. Heck, S. A. Maier "Plasmonic Nanoantennas: Fundamentals and Their Use in Controlling the Radiative Properties of Nanoemitters" Chemical Review 2011, 111, 6, 3888-3912.

[9] Z. Zhu, S. Joshi, B. Pelz, G. Moddel "Overview of optical rectennas for solar energy harvesting" SPIE Solar Energy Technology - International Society for Optics and Photonics 2013.

[10] P. Biagioni, J. S. Huang, B. Hecht "Nanoantennas for visible and infrared radiation" Report on Progress in Physics 75 (2012) 024402.

[11] A. E. Krasnok, et all.,"Optical nanoantennas",Physics Uspekhi 56, 539-564 (2013).

[12] L. Mescia, A. Massaro "New trends in Energy Harvesting from Earth Long-Wave Infrared Emission", Advanced in Material Sciences and Engineering (2014).

[13] A. M. A.Sabaawi, C. C. Tsimenidis, and B. S. Sharif "Overview of Nanoantennas for Solar Rectennas", chapter 11 Rectenna Solar Cells, Springer, pp.231-256, (2013).

[14] A. Abbas, M. El-Said, S. F. Mahmoud "Characteristics of an Optical Bowtie Nanoantenna" PIERS Proceedings, 2013.

[15] I. S. Maksymov, I. Staude, A. E. Miroshnichenko, Y. S. Kivshar "Optical Yagi-Uda nanoantennas" Nanophotonics $1,1,2012,65-81$.

[16] G. Abadal, J. Alda, J. Agustí "Electromagnetic Radiation Energy Harvesting - The Rectenna Based Approach" Chapter 5 ICT Energy Concepts Towards Zero Power Information and Communication Technology 2014.

[17] R. L. Olmon, M. B. Raschke "Antenna-load interactions at optical frequencies: impedance matching to quantum systems" Nanotechnology 23, 44 (2012).

[18] S. Grover, G. Moddel "Applicability of Metal/Insulator/Metal (MIM) Diodes to Solar Rectennas" IEEE Journal of Photovoltaics, vol. 1, n. 1, July 2011.

[19] A. E. Krasnok, et al. "All-Dielectric Optical Nanoantennas", Progress in Compact Antennas, Intech, p.143-175 (2014).

[20] D. W. Sciama "Modern cosmology", Cambridge University Press, 1971

[21] G. A.E. Vandenbosch, Z. Ma, "Upper bounds for the solar energy harvesting efficiency of nano-antennas", Nano Energy (2012) 1, 494-502.

[22] Da K. Q. da Costa, V. A. Dmitriev, "Analysis of modified Bowtie Nanoantennas in the excitation and emission regimes", Journal of Microwaves, Optoelectronics and Electromagnetic Application, Vol. 10, No. 1, (2011). 